\title{
A Unified Process for the Evaluation of the Zeros of Polynomials over the Complex Number Field
}

\author{
By John I. Derr \\ Summary
}

Newton's method for the evaluation of the zeros of analytic functions is generalized by the recurrence relation

$$
z_{i+1}=z_{i}-\left(k_{i}-l_{i}\right) f^{\left(l_{i}\right)}\left(z_{i}\right) / f^{\left(l_{i}+1\right)}\left(z_{i}\right),
$$

where $k_{i}$ and $l_{i}$ are determined so that ideally $l_{i}+1=k_{i}=k$ in the vicinity of a zero of order $k$. The process so defined is a second-order one and yields accurate approximations to zeros for all values of $k$, provided $l_{i}+1=k_{i}=k$.

1. Introduction. Consider the polynomial equation $f(z)=0$ in a complex variable $z$, where $f(z)$ has complex coefficients. To solve this equation by Newton's method one uses the conventional recurrence relation

$$
z_{i+1}=z_{i}+(\Delta z)_{i}, \text { where }(\Delta z)_{i}=-f\left(z_{i}\right) / f^{\prime}\left(z_{i}\right) .
$$

Bodewig [1] has shown that Newton's method is a second-order process if and only if the sequence $\left\{z_{i}\right\}$ converges to a simple zero of $f(z)$. He has shown further that the recurrence relation

$$
z_{i+1}=z_{i}+(\Delta z)_{i}, \text { where }(\Delta z)_{i}=-k f\left(z_{i}\right) / f^{\prime}\left(z_{i}\right),
$$

defines a second-order process whenever $\left\{z_{i}\right\}$ converges to a zero $\gamma$ of $f(z)$ of multiplicity $k$.

The process defined by equation (2) leaves two problems unsolved: (i) In practice $k$ and $\gamma$ are usually not known, ${ }^{*}$ (ii) The ultimate accuracy of the approximation to $\gamma$ is limited by the fact that $f\left(z_{i}\right), f^{\prime}\left(z_{i}\right) \rightarrow 0$ as $z_{i} \rightarrow \gamma$, provided $k \geqq 2$.

The unified process, which was developed to meet both of these problems, is characterized by the recurrence relation

$$
z_{i+1}=z_{i}-\left(k_{i}-l_{i}\right) f^{\left(l_{i}\right)}\left(z_{i}\right) / f^{\left(l_{i}+1\right)}\left(z_{i}\right),
$$

together with an explicit method for determining $l_{i}$ and $k_{i}$ which will be described in $\$ 2$. Note that (3) follows from (2) if $k_{i}=k$ and $f\left(z_{i}\right)$ is replaced by $f^{\left(l_{i}\right)}\left(z_{i}\right)$. Hence the unified process is a second-order process provided $0 \leqq l_{i}<k_{i}=k$. This justification of the unified process as a second-order process is the principal service provided by (2).

Received 9 Apr., 1957; revised 21 July, 1958.

* R. A. Brooker [2] describes a modification of (1) in which $k$ is approximated by the smallest positive integer $l$ such that

$$
\left|f\left[z_{i}-l f\left(z_{i}\right) / f^{\prime}\left(z_{i}\right)\right]\right|<\left|f\left[z_{i}-(l+1) f\left(z_{i}\right) / f^{\prime}\left(z_{i}\right)\right]\right|,
$$

provided $\left|f\left(z_{i+1}\right)\right|<\left|f\left(z_{i}\right)\right|$.

$\dagger$ The author is indebted to the referee for pointing out this simple connection between (2) and (3). This connection has influenced considerably the presentation of the first two sections. 
The principal result of this paper is the formulation of a method for determining $l_{i}$ and $k_{i}$ in such a way that $l_{i} \rightarrow k-1$ in a certain sense as $z_{i} \rightarrow \gamma$. In the standard application of Newton's method to a polynomial with multiple zeros the Euclidean algorithm would first be applied to eliminate the multiple zeros. (See [3].) This greatest common divisor process is, however, totally foreign to Newton's method, whereas our determination of $l_{i}$ and $k_{i}$ is intimately related to the computation of $f^{\left(l_{i}\right)}\left(z_{i}\right)$ and $f^{\left(l_{i}+1\right)}\left(z_{i}\right)$ in $(3)$. This close connection motivated the choice of the term "unified process."

2. The Unified Process. Let $f(z)=\sum_{i=0}^{n} c_{i} z^{i}$, where $n \geqq 1, c_{n}=1$, and $c_{i}$ is a complex number for $i=0,1, \cdots, n$. Throughout this section we shall assume that $\gamma$ is a zero of $f(z)$ of multiplicity $k$. Thus we can write $f(z)=(z-\gamma)^{k} q(z)$, where $q(\gamma) \neq 0$.

LEMMA 1.

$$
\frac{f^{m}(z)}{f^{m+1}(z)}=\frac{(z-\gamma)}{k-m}+0\left[(z-\gamma)^{2}\right]^{*} \quad \text { for } \quad m=0,1, \cdots, k-1 .
$$

Bodewig [1] obtains the same result for $m=0$. The lemma follows then by application of that result with $f(z)$ replaced by $f^{(m)}(z)$. From Lemma 1 (with $m=l_{i}$ ) equation (3) becomes $z_{i+1}=z_{i}-\left(k_{i}-l_{i}\right)\left[\left(z_{i}-\gamma\right) /\left(k-l_{i}\right)+0\left[\left(z_{i}-\gamma\right)^{2}\right]\right]$. So if $k_{i}=k$ we have $z_{i+1}=\gamma+0\left[\left(z_{i}-\gamma\right)^{2}\right]$. This result shows that equation (3) does define a second-order process when $0 \leqq l_{i}<k_{i}=k$. The main application of Lemma 1 will occur below in the determination of $k_{i}$.

Suppose that $z_{i}$ is a "close approximation" to $\gamma$. Let $\eta$ be a fixed, sufficiently small positive number. Then $l_{i}$ is defined as the smallest non-negative integer such that $\left|f^{\left(l_{i}+1\right)}\left(z_{i}\right)\right| \geqq \eta$.

Let us assume for the moment that $l_{i}>0$ if and only if $k>1$. We will justify this assumption below in Lemma 2. Then Lemma 1, when applied for $m=l_{i}$ and $l_{i}-1$ yields

$$
\left.\left(k-l_{i}+1\right)\left[\frac{f^{\left(l_{i}-1\right)}}{f^{\left(l_{i}\right)}}\right]\left(z_{i}\right)=\left(k-l_{i}\right)\left[\frac{f^{\left(l_{i}\right)}}{f^{\left(\bar{l}_{i}+1\right)}}\right]\left(z_{i}\right)+0\left[\left(z_{i}-\gamma\right)^{2}\right)\right] .
$$

The preceding equation suggests that we let $k_{i}=l_{i}+\hat{x}-1$ where $\hat{x}$ is the closest integer to

$$
x=\frac{f^{\left(l_{i}\right)}\left(z_{i}\right) / f^{\left(l_{i}+1\right)}\left(z_{i}\right)}{f^{\left(l_{i}\right)}\left(z_{i}\right) / f^{\left(l_{i}+1\right)}\left(z_{i}\right)-f^{\left(l_{i}-1\right)}\left(z_{i}\right) / f^{\left(l_{i}\right)}\left(z_{i}\right)} .
$$

We can now give an explicit description of how the unified process operates to extract an arbitrary and unknown zero of $f(z)$. Let $\eta$ and the starting value $z_{1}$ be given. Then $z_{i+1}$ is determined recursively from $z_{i}$ by means of equation (3), where for each $i, l_{i}$ is the first non-negative integer to satisfy $\left|f^{\left(l_{i}+1\right)}\left(z_{i}\right)\right| \geqq \eta$, and $k_{i}$ is 1 or $l_{i}+\hat{x}-1$ depending on whether $l_{i}$ is respectively zero or positive. To obtain $\hat{x}$, round $x$ as given in (4) to the nearest integer.

We know that the unified process defines a second-order process if

$$
0 \leqq l_{i} \leqq k_{i}-1=k-1 \text {. }
$$

${ }^{*} \mathrm{O}(x)$ is a quantity which vanishes at least as fast as $x$, i.e., $|0(x) / x|$ is bounded as $x \rightarrow 0$. 
In looking ahead to the next section it is evident from accuracy considerations that in practice we would prefer that $l_{i}=k_{i}-1=k-1$; for note that then equation (3) becomes

$$
z_{i+1}=z_{i}-f^{(k-1)}\left(z_{i}\right) / f^{(k)}\left(z_{i}\right), \text { and } f^{k}(\gamma) \neq 0 .
$$

The following lemma shows that the situation where $l_{i}=k-1$ is always obtained as soon as $z_{i}$ is close enough to $\gamma$.

Lemma 2. Suppose $z_{i} \rightarrow \gamma$, where $\left\{z_{i}\right\}$ is determined by the unified process. Then there exist positive integers $N_{1}, N_{2}\left(N_{1} \leqq N_{2}\right)$, such that $0 \leqq l_{i} \leqq k-1$ for $i \geqq N_{1}$ and $l_{i}=k-1$ for $i \geqq N_{2}$.

The proof of Lemma 2 follows from the continuity of $f^{(m)}(z)$ for $m=0,1, \cdots, k$; the fact that $f^{(k)}(\gamma) \neq 0$ while $f^{(m)}(\gamma)=0$ for $m=0,1, \cdots, k-1$; and finally the fact that $0 \leqq m \leqq l_{i}$ implies $\left|f^{(m)}\left(z_{i}\right)\right|<\eta$.

The following restriction on $\eta$ follows immediately.

Condition oiv $\eta$. Suppose $\left\{z_{i}\right\}$ is determined by the unified process. Then $z_{i} \rightarrow \gamma$ implies that $\left|f^{(k)}(\gamma)\right| \geqq \eta$, where $k$ is the multiplicity of $\gamma$.

Example 1. Suppose, for convenience, that $\eta<.01$. Let

$$
f(z)=(z-1)^{2}(z+1)^{2}=z^{4}-2 z^{2}+1 .
$$

Let $z_{1}=2^{n}$. It follows that $\Delta z=-f / f^{\prime}=-(z-1 / z) / 4=-z / 4+0(1 / z)$. Then $z_{2}>2^{n-1}$, and by induction $z_{n}>2$.

Also, $f(1+\delta)=4 \delta^{2}+4 \delta^{3}+\delta^{4}$, and $f^{\prime}(1+\delta)=8 \delta+12 \delta^{2}+4 \delta^{3}$. Therefore, $\Delta z=-\delta / 2+0\left(\delta^{2}\right)$. It is easy to verify that $1+\eta \leqq z_{N_{1}} \leqq 2$ for some $N_{1}>n$. Thus $f^{\prime}\left(z_{N_{1}}\right) \geqq \eta$ and we have $l_{N_{1}}+1=1<k$. However, we do indeed have $0 \leqq l_{i} \leqq k-1$ for $i \geqq N_{1}$.

Next choose $N_{2} \geqq N_{1}$ such that $i>N_{2}$ implies $z_{i}<1+\eta^{2}$. Then $i>N_{2}$ implies $\left|f^{\prime}\left(z_{i}\right)\right|<\left|f^{\prime}\left(1+\eta^{2}\right)\right|<\eta / 2$. Therefore, $l_{i} \geqq 1$. Since

$$
f^{\prime \prime}(1+\delta)=8+24 \delta+12 \delta^{2}
$$

it follows that $l_{i}+1=k$ for all $i>N_{2}$. Thus $N_{1}$ and $N_{2}$ satisfy the conditions of Lemma 2.

In summary the behavior of the process for large real $z$ in this example is that which would be expected for a 4 th order multiple root at the origin, for with

$$
f(z)=z^{4}
$$

we get $f(z) / f^{\prime}(z)=z / 4$ and $\left|f^{\prime}(z)\right|>\eta$; and we have $\Delta z=-z / 4+0(1 / z)$. As $z_{i} \rightarrow 1$, however, the behavior approaches that predicted by Lemma 1 near a double root at $z=1$, i.e., $(z-\gamma) /(k-m)=(z-1) / 2$, when $m=0$. We have seen that $\Delta z=-(z-1) / 2+0\left[(z-1)^{2}\right]$.

In this section we have considered the unified process in an abstract setting in the sense that we have passed to the limit and employed complex numbers in the ordinary fashion. By so doing we have been able to present the basic facts about the process, especially as regards convergence, in a form unencumbered by the complications we shall encounter in the next section. There we shall introduce an approximate convergence for a class of complex numbers whose real and imaginary parts have decimal expansions of bounded length. We can then indicate precisely how the second problem mentioned in $\$ 1$ is relevant. 
It is clear that we have already solved both of the problems set forth in $\S 1$ in the sense that if we have convergence at all, then the convergence is of the second order and eventually the situation in (5) holds. As to when the process does converge, we refer the reader to the existence theorems for Newton's method given by Ostrowski [8]. However, it is not obvious how anything useful (in a practical sense) can result from a non-trivial adaptation of such theorems to the unified process. On the other hand see Brooker [2] for an example where Newton's method does not converge.

3. Numerical Application. From now on we consider only those complex numbers whose real and imaginary parts contain $p$ decimal digits. Consistent with our "rounded-off" numbers we now define the numerical convergence of truncated sequences $\left\{z_{i}\right\}$ of such numbers. Let $\varepsilon>0$ be fixed and let $M$ be a positive integer, which depends only on $\left\{z_{i}\right\}$. We say that $\left\{z_{i}\right\}$ converges if there exists a positive integer $N \leqq M$ such that $\left|(\Delta z)_{N}\right| \leqq \varepsilon\left|z_{N}\right|$. We also assume without loss of generality that $f(0) \neq 0$, i.e., $c_{0} \neq 0$.

Situations producing non-convergence usually fall into one of two classes: (i) $z_{i}$ occasionally lies in a sufficiently small neighborhood of a root of $f^{\prime}(z) / f(z)$. This occurs frequently, since by Lucas' theorem any convex polygon containing all of the roots of $f(z)$ contains all of the roots of $f^{\prime}(z)$. (See [5].) (ii) A subsequence of $\left\{z_{i}\right\}$ is cyclic.

Class (ii) may occur due to round-off error, e.g., because of lack of precision in the coefficients of $f(z)$ or because of multiple roots if $k_{i}<k$. In addition there are bona fide situations like the one referred to at the end of $\$ 2$, where accuracy considerations play no part. We employ a systematic method for selecting a new value for $z_{1}$ whenever non-convergence occurs.

We now modify our method for determining $l_{i}$ and $k_{i}$ so as to be consistent with our approximate arithmetic. We first define $l_{i}$ to be the smallest non-negative integer (if such exists) such that

(i) $\left|q_{l_{i+1}}\left(z_{i}\right)\right| \geqq \eta\left|q_{l_{i+1}}(0)\right|$ and

(ii) $\left|q_{l_{i-1}}\left(z_{i}\right)\right|<\eta^{2}\left|q_{l_{i-1}}(0)\right|$, whenever $l_{i}>0$.

In the above expressions $\left\{q_{m}(z)\right\}$ are the quotient polynomials which are obtained by dividing $f(z)$ successively by $z-z_{i}$. More precisely, define recursively

$$
f(z)=q_{0}(z)=\left(z-z_{i}\right) q_{1}(z)+f\left(z_{i}\right)
$$

and $q_{m}(z)=\left(z-z_{i}\right) q_{m+1}(z)+q_{m}\left(z_{i}\right)$ for $m=1,2, \cdots, l_{i}$. Note that in general $f^{m}\left(z_{i}\right)=m ! q_{m}\left(z_{i}\right)$. If no such $l_{i}<n$ exists, replace $z_{i}$ by $z_{i}-(\Delta z)_{i-1} / 2$.

Condition (ii) has been added to the original definition of $l_{i}$ in order to help detect non-convergence class (i) and to separate closely bunched zeros. Example 2 will show how $\log _{10}\left|q_{m}(0) / q_{m}\left(z_{i}\right)\right|$ gives a measure of the number of significant digits lost in the evaluation of $f^{(m)}\left(z_{i}\right)$. We also encounter in Example 2 a limiting situation where two zeros are just close enough so that condition (ii) fails to separate them.

The determination of $k_{i}$ differs (when $l_{i}>0$ ) only by the manner in which $\hat{x}$ is determined. As regards $\hat{x}$, let $\delta$ be a fixed small positive number. Then 
(i) Set $\hat{x}=j$ if $x$ is well-defined by (4) and there exists an integer $j$,

$$
2 \leqq j \leqq n-l_{i}+1
$$

such that $|x-j|<\delta$.

(ii) Set $\hat{x}=2$ otherwise.

Note that (ii) results in setting $k_{i}=l_{i}+1$. Recall that $k_{i}=\hat{x}+l_{i}-1$ when $l_{i}>0$.

As motivation for (i) we know that if $\gamma$ is a multiple root and $z_{i}$ is so close to $\gamma$ that $l_{i}>1$, then $x$ should be close to an integer which is bounded below by 2 and above by $n-l_{i}+1$. When the corresponding smallest value of $\hat{x}$-namely, $\hat{x}=2$ -is taken on, then $l_{i}+1=k_{i}=k$, while $k_{i} \leqq n$ implies

$$
\hat{x}=k_{i}-l_{i}+1 \leqq n-l_{i}+1 \text {. }
$$

We make the assumption that case (ii) reflects a loss of significant digits in the computation of $f^{\left(l_{i}-1\right)}\left(z_{i}\right) / f^{\left(l_{i}\right)}\left(z_{i}\right)$, due to the nearness of $z_{i}$ to a multiple root $\gamma$. The assumption is biased toward the selection of multiple roots as Example 2 indicates. In the discussion of Example 2 a modification of (ii) is described which is biased toward the separation of roots.

Example 2. Suppose $\eta<.1$ and consider

$$
f(z)=z^{2}-(2+\eta) z+(1+\eta)=(z-1)(z-1-\eta) .
$$

Suppose $z_{N}=1$. Then $q_{1}(0)=-1-\eta$ and $f^{\prime}\left(z_{N}\right)=-\eta$. Hence,

$$
\left|q_{1}\left(z_{N}\right)\right|<\eta\left|q_{1}(0)\right|
$$

and $l_{N}>0$. Further, $q_{2}(0)=1$ implies $l_{N}=1$.

Since $f^{\left(l_{N}-1\right)}\left(z_{N}\right)=0, x=1$ and $k_{N}=l_{N}+1$. It follows that $(\Delta z)_{N}=\eta / 2 .^{*}$ But $q_{0}(1+\eta / 2)=-\eta^{2} / 4$ and $f^{\prime}(1+\eta / 2)=0$. Therefore, $l_{N+1}=1$ and

$$
(\Delta z)_{N+1}=0 \text {. }
$$

As a result $1+\eta / 2$ is identified as a double root of $f(z)$. Observe that the final step in the computation of $q_{0}(1+\eta / 2)$ is the addition of $q_{0}(0)$ and $-1-\eta-\eta^{2} / 4$.

The example also shows that sequences $\left\{z_{i}\right\}$ can converge to numbers which differ from roots of $f(z)$ by more than could be attributed to round-off error. In particular, the distinct roots 1 and $1+\eta$ are treated as one double root equal to the mean value of 1 and $1+\eta$. We shall indicate how this situation may generalize. By making use of the following lemma it suffices to produce a set of roots and a value of $z_{N}$ such that $l_{i}=n-1$.

LEMMA 3. Let $f(z)$ be an arbitrary polynomial of degree $n$ having roots $\gamma_{1}, \cdots$, $\gamma_{h}$ with multiplicities $m_{1}, \cdots, m_{h}$, respectively. Then for every complex number $z_{0}$ we have

$$
z_{0}-f^{(n-1)}\left(z_{0}\right) / f^{(n)}\left(z_{0}\right)=\sum_{i=1}^{h} m_{i} \gamma_{i} / n .
$$

In particular, if $f(z)=(z-\gamma)^{n}$, then $z_{0}-f^{n-1}\left(z_{0}\right) / f^{n}\left(z_{0}\right)=\gamma$. Here we have a trivial case of "infinite order" of convergence, i.e., when $l_{i}=n-1$, the problem

* Note that if the determination of $k_{i}\left(l_{i}>0\right)$ were modified so that when $|x-1|<\delta$, $k_{i}=l_{i}$ and $l_{i}$ is decreased by 1 , then we would have $l_{N}=0$ and $(\Delta z)_{N}=0$. 
of finding $\gamma$ under the unified process generalizes the trivial problem of solving a linear equation by Newton's method.

We know that for every $\eta$ there exist polynomials such that distinct roots will be identified as multiple roots. Clearly, in order to remedy this situation in any particular case we must decrease $l_{i}$ and hence $\eta$. However, we are at one side of a dilemma. For consider the case where $\gamma$ actually is a multiple root. Then if $\eta$ is sufficiently small, we must have $z_{i}$ very close to $\gamma$ before $l_{i}$ can exceed 0 -which means that as $\eta$ is decreased, multiple roots tend more to be split into roots of lower multiplicities with their maximum accuracy limited to $p /\left(k-k_{i}+1\right)$ digits. Recall from Example 1 that $\Delta z=-(z-1) / 2+0\left[(z-1)^{2}\right]$ for $z$ near 1 . Thus $z=1+\eta$ would satisfy our numerical convergence criterion if $\eta \leqq \varepsilon$ and be identified as a simple zero.

The fact that $z=1+\eta$ would be determined to be within $\varepsilon$ distance of the double root at 1 , although of the wrong multiplicity, would probably suffice for most practical cases. However, there do exist situations where the diagnosis of multiplicity is important in its own right, e.g., $\int_{0}^{1} d x / \sqrt{x(x-\varepsilon)}$ converges, whereas $\int_{0}^{1} d x / \sqrt{x^{2}}$ does not. Moreover, in addition to such special cases, the best results are usually obtained when $l_{i}+1=k_{i}=k$.

We have observed that $l_{i}$ is a nondecreasing function of $\eta$. Observe, however, that the approximate solutions obtained by the unified process do not depend continuously on $\eta$, since $l_{i}$ and $k_{i}$ are integer-valued functions. We have also seen that an optimum value of $\eta$ depends strongly upon the distribution of the roots near $z_{i}$. Roughly speaking, if several distinct roots are bunched together, $\eta$ should be "small."* On the other hand, if only a multiple root is near $z_{i}$, then $\eta$ should be "large." For general calculations we recommend that $\eta=\sqrt{\varepsilon}$ and $1 / \varepsilon \leqq 10^{p-2}$.

4. Computational Examples. In this section we shall extend the discussion of the preceding examples. In particular we shall record and interpret some solutions which were obtained on The RAND Corporation JOHNNIAC computer using nine-digit floating decimal (scientific notation) arithmetic $(p=9)$ with $\varepsilon=10^{-7}$, and $\delta=10^{-3}$. We illustrate the sensitivity of the process to $\eta$ by considering $\eta=$ $10^{-3}$ and $\eta=10^{-4}$.

Example 3. We consider Example 1 again, i.e., $f(z)=z^{4}-2 z^{2}+1$. With $\eta=10^{-4}$ the pair $\dagger$ of complex conjugate roots, $1 \pm i\left(.663098208 \cdot 10^{-4}\right)$, and the associated reduced polynomial, $q(z)=z^{2}+2 z+1$, were first obtained. Note that in addition to the fact that the double root +1 was approximated by a pair of simple roots, the modulus of the relative error substantially exceeds $\varepsilon$.

Recall that $f(1+\theta) \sim 4 \theta^{2}$ and $f^{\prime}(1+\theta) \sim 8 \theta$ as $\theta \rightarrow 0$. Thus at

$$
z_{i}=1+i\left(.663098208 \cdot 10^{-4}\right) \text {, }
$$

we have $f\left(z_{i}\right) \doteq-2 \cdot 10^{-8}$ and $f^{\prime}\left(z_{i}\right) \doteq i\left(.5 \cdot 10^{-3}\right)$. However, due to round-off error the evaluation of $f\left(z_{i}\right)$ yields zero. Therefore, $(\Delta z)_{i}=0$, since $\left|f^{\prime}\left(z_{i}\right)\right|>\eta$.

* In the next section we shall show that this is not always the case.

$\dagger$ The roots were actually obtained in succession. When a root is determined the reduced quotient polynomial $q_{k_{i}}(z)$ replaces $f(z)$. 
On the other hand, with $\eta=10^{-3}$ we obtained the double roots

$$
1+i\left(.000000015 \cdot 10^{-8}\right)
$$

and $-.999999998+i\left(.753350000 \cdot 10^{-8}\right)$. Here the relative error is less than $\varepsilon$ in either case. The first double root was obtained because now $.5 \cdot 10^{-3}<\eta$.

Example 4. The polynomial in Example 2 is now generalized to the class of polynomials of the form $z^{2}-(2+\theta) z+(1+\theta)$. The results tabulated immediately below were obtained with $\eta=10^{-4}$.

\begin{tabular}{lll}
\multicolumn{1}{c}{$\theta$} & \multicolumn{1}{c}{ Roots } & $\mid$ Error $\mid$ \\
.1 & $1.09999996-i\left(.000000001 \cdot 10^{-8}\right)$ & .00000004 \\
.01 & $1.00000004-i\left(.490000000 \cdot 10^{-9}\right)$ & \\
& $1.00999900-i\left(.000000001 \cdot 10^{-16}\right)$ & .000001 \\
.001 & $1.00000100+i\left(.200000000 \cdot 10^{-18}\right)$ & \\
& $1.00099296-i\left(.000000002 \cdot 10^{-12}\right)$ & .00000704 \\
.0001 & $1.00000704+i\left(.100000000 \cdot 10^{-14}\right)$ & .00005 \\
.00001 & $1.00005000 \quad$ (double root) & .000005
\end{tabular}

With $\eta=10^{-3}$ and everything else unchanged, the results obtained were the same except that for $\theta=.001$ we obtained 1.00050000 as a double root with an error of .0005 in magnitude.

If $z=1+\Delta$, we have $f(z)=\Delta(\Delta-\theta)$ and $f^{\prime}(z)=2 \Delta-\theta$. With $l_{i} \equiv 0$ it is clear that as $z_{i} \rightarrow 1+\theta, \Delta_{i} \rightarrow \theta$ and

$$
(\Delta z)_{i}=\frac{\Delta_{i}\left(\theta-\Delta_{i}\right)}{\Delta_{i}+\left(\Delta_{i}-\theta\right)}=\left(\theta-\Delta_{i}\right)+0\left[\left(\theta-\Delta_{i}\right)^{2}\right] .
$$

Such a situation can always be arranged if $\eta<\theta$, e.g., if $\eta=10^{-4}$ and $\theta \geqq 10^{-3}$. Under these circumstances $\left\{z_{i}\right\}$ converges numerically as soon as $\left|\theta-\Delta_{i}\right| \leqq \varepsilon$. $^{*}$ However, since we are using nine-digit arithmetic, $(\Delta z)_{i}$ cannot be computed with enough accuracy to make the comparison with $\varepsilon$ unless $\left|\theta-\Delta_{i}\right| \geqq \varepsilon /\left(10 \Delta_{i}\right)$; for otherwise $\left|\Delta_{i}\left(\Delta_{i}-\theta\right)\right|$ has less than one significant digit. Therefore, $1 /\left(10^{8} \theta\right)$ yields a close estimate for the magnitude of relative error. The estimate accounts for the increase in error as $\theta$ decreases from .1 to .001 for the results tabulated above.

We conclude the discussion of Example 4 by making several remarks which have general implications.

(i) When roots are bunched close together, the magnitude of relative error can exceed $\varepsilon$ even when the multiplicity is correct.

(ii) If the roots are sufficiently close together, then the relative error will be decreased if the multiplicity is determined at certain values greater than the true multiplicity. In Example 4, 1 and $1+\theta$ will be sufficiently close as soon as

$$
1 /\left(10^{8} \theta\right)=\theta / 2 \text {, i.e. } \theta=\sqrt{2} \cdot 10^{-4} \text {. }
$$

5. Conclusion. Notwithstanding the tremendous effort which has been expended on the problem over the years, the recent appearance of the high-speed computer

* The argument for the remainder of this section is not precise, but only as a result of secondary considerations. 
and the corresponding increase in size of the practical problems which can be attacked have created new demands for computational methods. For example, the methods advocated as late as 1952 by Olver [7], being non-iterative in character, are quite different from those which are considered here. One explanation for the difference in emphasis might be that a computer is very efficient at executing repetitively a simple sequence of operations. For the unified process a great bulk of the computation involves one basic operation, namely, synthetic division performed with complex numbers. When applied to a computer the unified process is flexible and automatic and can be further controlled by manipulating sensitive quantities such as $\eta, \delta, \varepsilon$, and $z_{1}$.

For comparison purposes we consider two methods which have proved useful for digital computers. Under certain circumstances each method evaluates roots with about one-half the effort required by the unified process. However, both methods require special preparation in the case of multiple roots.

Hitchcock's method [4] requires that $f(z)$ have real coefficients and utilizes the resulting decomposition of $f(z)$ over the reals into linear and quadratic factors to avoid complex arithmetic in the synthetic divisions. Otherwise, the method is essentially Newton's method.

Muller's method does not require the evaluation of $f^{\prime}\left(z_{i}\right)$. When the degree of $f(z)$ is large, a saving of close to $50 \%$ results. The order of convergence of the process is 1.84 for simple roots and 1.23 for double roots. Apparently, no improvement over Newton's method obtains for multiple roots whose order exceeds two.

Most of our discussion remains valid for arbitrary analytic functions. \$2 goes through intact, but the application described in $\$ 3$ requires some modifications. Moreover, for the method to be useful in practice the class of functions considered should be restricted to those which have a non-empty, finite set of zeros and for which the derivatives are easy to compute.

Rand Corporation, Santa Monica, California

1. E. Bodewig, "On Types of Convergence and on the Behavior of Approximations in the Neighborhood of a Multiple Root of an Equation," Quarterly of Applied Mathematics, Volume VII, p. 325-333.

2. R. A. Brooker, "The Solution of Algebraic Equations on the EDSAC," Proceedings of the Cambridge Philosophical Society, Vol. 48, 1952.

3. SAUL Gorn, "Real Solutions of Numerical Equations by High-Speed Machines," BRL Report No. 966, October, 1955.

4. Frank L. Hitchcock, "An Improvement on the G.C.D. Method for Complex Roots," Journal of Math and Physics, v. 23, 1944.

5. Morris Marden, "The Geometry of the Zeros of a Polynomial in a Complex Variable," American Math. Society Survey III, N. Y., 1949.

6. David E. Muller, "A Method for Solving Algebraic Equations Using an Automatic Computer, Mathematical Tables and Oiher Aids to Computation," v. 10, 1956.

7. F. W. J. Olver, "Evaluation of Zeros of High-Degree Polynomials," Philosophical Transactions of the Royal Society of London, Volume 244, April 3, 1952.

8. A. N. Ostrowski, "Lectures on Solutions of Equations and Systems of Equations," NBS Working Paper, June 11, 1952. 